\title{
Single-step genomic evaluation using multitrait random regression model and test-day data
}

\author{
M. Koivula, ${ }^{* 1}$ I. Strandén, ${ }^{*}$ J. Pösö,† G. P. Aamand,† and E. A. Mäntysaari \\ *Natural Resources Institute Finland (Luke), Green Technology, 31600 Jokioinen, Finland \\ †Faba Co, 01301 Vantaa, Finland \\ łNAV Nordic Cattle Genetic Evaluation, Agro Food Park 15, 8200 Aarhus N, Denmark
}

\section{ABSTRACT}

The objectives of this study were to evaluate the feasibility of use of the test-day (TD) single-step genomic BLUP (ssGBLUP) using phenotypic records of Nordic Red Dairy cows. The critical point in ssGBLUP is how genomically derived relationships $(\mathbf{G})$ are integrated with population-based pedigree relationships $(\mathbf{A})$ into a combined relationship matrix $(\mathbf{H})$. Therefore, we also tested how different weights for genomic and pedigree relationships affect ssGBLUP, validation reliability, and validation regression coefficients. Deregressed proofs for 305-d milk, protein, and fat yields were used for a posteriori validation. The results showed that the use of phenotypic TD records in ssGBLUP is feasible. Moreover, the TD ssGBLUP model gave considerably higher validation reliabilities and validation regression coefficients than the TD model without genomic information. No significant differences were found in validation reliability between the different TD ssGBLUP models according to bootstrap confidence intervals. However, the degree of inflation in genomic enhanced breeding values is affected by the method used in construction of the $\mathbf{H}$ matrix. The results showed that ssGBLUP provides a good alternative to the currently used multistep approach but there is a great need to find the best option to combine pedigree and genomic information in the genomic matrix.

Key words: genomic evaluation, single step, testday model, Nordic Red Dairy cow, single-step genomic BLUP (ssGBLUP)

\section{INTRODUCTION}

Most genomic evaluations are based on multi-step approach that requires (1) calculation of traditional EBV without genomic information; (2) extraction of pseudo-observations, typically either daughter yield

Received October 14, 2014.

Accepted December 16, 2014

${ }^{1}$ Corresponding author: minna.koivula@luke.fi deviations (DYD) or deregressed EBV (deregressed proofs; DRP); and (3) genomic model for prediction of direct genomic values (DGV; VanRaden, 2008; Hayes et al., 2009; VanRaden et al., 2009). Genomic evaluations can be further improved by combining the DGV and information from traditional EBV (e.g., VanRaden, 2008) to yield genomic enhanced breeding values (GEBV).

The multi-step approach to calculate GEBV has an inherent problem. First, the parent averages (PA) of progeny of genomically selected animals do not automatically include genomic information. Second, when animals are selected by their GEBV, the future estimation of unbiased EBV becomes difficult because genomic information is not taken into account in the traditionally calculated EBV. Moreover, genomic selection using the multi-step approach is complex and includes several approximations, all of which reduce accuracy and can inflate the resultant GEBV. None of these issues applies to the single-step approach.

Single-step evaluation (single-step genomic BLUP; ssGBLUP) is a unified approach to calculate GEBV. The ssGBLUP combines phenotypic records, pedigree information, and genomic information optimally in calculation of GEBV (Misztal, et al., 2009; Aguilar et al., 2010; Christensen and Lund, 2010). The approach integrates the pedigree relationship matrix $\mathbf{A}$ and genomic relationship matrix $\mathbf{G}$ into a single $\mathbf{H}$ matrix, which replaces the traditional relationship matrix $\mathbf{A}$ in the mixed-model equations (Legarra et al., 2009; Misztal et al., 2009; Christensen and Lund, 2010). To date, the single-step approach has been rated computationally demanding with large data sets and multi-trait analysis (Su et al., 2012). However, ssGBLUP has been successfully applied, for example, for final scores of over 6 million Holsteins with greater accuracy than that of a multi-step procedure (Aguilar et al., 2010), and in a multi-trait national genomic evaluation for type traits in US Holsteins (Tsuruta et al., 2011). Performance of ssGBLUP has also been evaluated in other species. Chen et al. (2011) used ssGBLUP to analyze 3 traits in 2 separately selected lines of chickens, and Forni et 
al. (2011) used ssGBLUP to analyze litter size in pigs. Thus, despite its high computational requirements, the single-step method is suitable for large multi-trait analyses. The critical issue with ssGBLUP is compatibility between the marker-based relationship matrix and the pedigree-based relationship matrix for genotyped animals. Applications of the first unified approaches for merging information from animals with or without genotypes by combining the $\mathbf{A}$ matrix with the $\mathbf{G}$ matrix resulted in biased GEBV (e.g., Meuwissen et al., 2011). Since then, it has been demonstrated that accuracy of prediction can be improved and bias reduced by adjusting the $\mathbf{G}$ matrix toward their expected values in the $\mathbf{A}$ matrix to decrease the scaling problem (e.g., Vitezica et al., 2011; Christensen et al., 2012).

A random regression test-day (TD) model is currently used for the official Nordic genetic evaluation of production (Lidauer et al., 2015) in Nordic Red Dairy Cattle (RDC). As more selection decisions are made using genomic information, it is becoming essential that all genomic information is included in national evaluations. The objectives of this study were to evaluate the feasibility of the large random regression TD ssGBLUP, and to estimate the accuracy of GEBV when using this model. We also tested how different combinations of the $\mathbf{A}$ and $\mathbf{G}$ matrices affect the bias and accuracy of GEBV in the TD ssGBLUP.

\section{MATERIALS AND METHODS}

All analyses used the data used in the official Nordic RDC milk production evaluations. The multiple-trait milk production evaluation includes TD records for milk, fat, and protein production. Production records from the first 3 lactations are in the same multiple-trait model. Each trait has random regression function for random genetic and permanent environmental effects. For more information, see Lidauer et al. (2015).

The routine full evaluation data from May 2014 for the RDC were obtained from the Nordic Cattle Genetic Evaluation (NAV; Aarhus, Denmark). For production traits, the TD data included 3.8 million cows with a total of 85 million records and 5.1 million animals in the Nordic RDC pedigree. To be able to validate the model, a reduced data set was extracted from the full data set, as follows: the last $4 \mathrm{yr}$ of observations were removed and the reduced data included 2.7 million cows with 72 million records. The reduced data set was used to solve GEBV and EBV for all animals in the pedigree, and the full data set was used to solve current EBV for testing purpose. The initial EBV from the reduced data set were denoted $\mathbf{E B V}_{\mathbf{r}}$. For the females without observations and bulls without daughters in reduced data, $\mathrm{EBV}_{\mathrm{r}}$ are hereafter referred to as parent average (PA). Comparing initial predictions from the reduced data set with those from the full data set allowed estimation of validation accuracy (Mäntysaari et al., 2010). The total number of equations in the reduced run was $217,370,251$, and in the full run 238,041,030.

The unified relationship matrix $\mathbf{H}$ in single-step evaluations defines the relationships among genotyped and nongenotyped animals. Although $\mathbf{H}$ can be expensive to compute, its inverse has a simple structure (Aguilar et al., 2010; Christensen and Lund, 2010):

$$
\mathbf{H}^{-1}=\mathbf{A}^{-1}+\left[\begin{array}{cc}
0 & 0 \\
0 & \mathbf{G}^{-1}-\mathbf{A}_{22}^{-1}
\end{array}\right],
$$

where $\mathbf{A}_{22}$ is the sub-matrix of the pedigree-based numerator relationship matrix $\mathbf{A}$ for the genotyped animals, and $\mathbf{G}$ is the relationship matrix constructed using genomic information. The $\mathbf{G}$ matrix had 15,148 genotyped RDC animals, of which 5,534 were bulls and 9,529 cows. The $\mathbf{G}$ matrix also included genotypes of animals without offspring or records. Genotypes were obtained from the Illumina Bovine SNP50 Bead Chip (Illumina, San Diego, CA). After application of exclusion criteria, 46,914 SNP markers on the 29 bovine autosomes were available for further analysis. The genotype file was the same as was used in official genomic evaluation of Nordic Cattle Genetic Evaluation in June 2014. Genotypes were used to form the raw G matrix with method 1 in VanRaden (2008). Before the matrices $\mathbf{G}$ and $\mathbf{A}_{22}$ were combined, the raw $\mathbf{G}$ matrix was scaled by scalar $t=\frac{\operatorname{tr}\left(\mathbf{A}_{22}\right)}{\operatorname{tr}(\mathbf{G})}$, where $t r$ is the trace of matrix. Thus, $\mathbf{G}$ has, on average, the same diagonals as the $\mathbf{A}_{22}$ matrix.

When the mixed-model equation for single-step is considered, the difference from the normal animal model is the matrix block $\mathbf{H}^{22}=\mathbf{A}^{22}+\mathbf{G}^{-1}-\mathbf{A}_{22}{ }^{-1}$ between genotyped animals. To improve the properties of the ssGBLUP, different weights in building the $\mathbf{H}^{22}$ matrix were tested. Aguilar et al. (2010) and Christensen and Lund (2010) noted that if not all genetic variance is accounted for by the SNP effects, the residual polygenic effect can be included in the model by changing the genomic matrix $\mathbf{G}$ and using $\mathbf{H}^{22}=\mathbf{A}^{22}+\mathbf{G}_{\mathrm{w}}^{-1}-\mathbf{A}_{22}^{-1}$, where $\mathbf{G}_{\mathrm{w}}=(1-\mathrm{w}) \mathbf{G}+\mathrm{w} \mathbf{A}_{22}$, and the constant $\mathrm{w}$ represents the proportion of polygenic variance not described by markers. So, the smaller $\mathrm{w}$, the more genetic variance that is attributed to genomic markers. We used 3 different proportions $\mathrm{w}$ $(\mathrm{w}=0.10, \mathrm{w}=0.15$, or $\mathrm{w}=0.20)$ in $\mathbf{G}_{\mathrm{w}}$. In Christensen et al. (2012), the optimal $\mathrm{w}$ was found to be 0.20 , al- 
though differences in accuracy with different $\mathrm{w}$ were small. In Misztal et al. (2010) and Tsuruta et al. (2011), the $\mathbf{H}^{22}$ matrix was further scaled to be $\mathbf{H}^{22}=\mathbf{A}^{22}+\tau \mathbf{G}_{\mathrm{w}}^{-1}-\omega \mathbf{A}_{22}^{-1}$. Misztal et al. (2013) suggested that optimal weights $\tau$ for $\mathbf{G}_{\mathrm{w}}^{-1}$ and $\omega$ for $\mathbf{A}_{22}^{-1}$ decrease the possible inflation of GEBV estimated by ssGBLUP. The parameters $\tau$ and $\omega$ scale the size of the genomic and pedigree relationships, respectively. The larger $\tau$ is, the less weight is given to $\mathbf{G}$, whereas larger values of $\omega$ decrease the importance of pedigree relationships and increase the importance of genomic relationships. We tested 4 combinations of these parameters. The first was the combination found best in Misztal et al. (2010): $\tau=1.5$ and $\omega=0.6$. In the other set ups, the weights were selected to be $\tau=1.6$ and $\omega$ $=0.5, \tau=1.6$ and $\omega=1.0$, or $\tau=1.0$ and $\omega=0.5$. In the tests with different $\tau$ and $\omega$, the proportion of polygenic variance was fixed to $\mathrm{w}=0.10$ in $\mathbf{G}_{\mathrm{w}}$. In the following, these different methods are referred to as follows: $\mathrm{w}_{20}, \mathrm{w}_{15}, \mathrm{w}_{10}, \tau_{1.6} \omega_{1.0}, \tau_{1.0} \omega_{0.5}, \tau_{1.6} \omega_{0.5}$, and $\tau_{1.5} \omega_{0.6}$. Note that the method using $\mathrm{w}=0.10$ corresponds to the situation where $\tau=1.0$ and $\omega=1.0$; however, we refer to this method as $\mathrm{w}_{10}$, instead of $\tau_{1.0} \omega_{1.0}$.

The analyses used the NAV routine EBV milk production evaluation model, in which a multiple-trait TD model is used to estimate EBV of milk, fat, and protein simultaneously. The GEBV were obtained from the TD ssGBLUP. Unknown parents by genetic groups and inbreeding coefficients were taken into account in the model. The mixed model equations were solved by MiX99 software using iteration on data and preconditioned conjugate gradients (PCG) iteration (Strandén and Lidauer, 1999). Computations for EBV and GEBV were very similar. Equations to solve EBV used inverse of $\mathbf{A}^{-1}$, which was replaced by alternative $\mathbf{H}^{-1}$ matrices in the single-step method for GEBV. In the PCG algorithm, the iteration involves multiplication of search direction vector $\mathbf{v}$ by the MME coefficient matrix. The implementation of the single-step method in MiX99 splits the required matrix multiplications into several steps. The first step is the multiplication of the least squares part of the coefficient matrix and the second is the product $\mathbf{H}^{-1} \mathbf{v}$. This is further divided into 2 steps. First, the product $\mathbf{A}^{-1} \mathbf{v}$ is computed directly by reading the pedigree file as is done in the traditional EBV calculation. Second, the product $\mathbf{H}^{22} \mathbf{v}$ is calculated by reading $\left[\mathbf{H}^{22}-\mathbf{A}^{22}\right]$ from a separate file during each PCG iteration cycle. Thus, the only additional work for the single step in solving MME is the matrix times vector product $\mathbf{H}^{22} \mathbf{v}$ in each PCG iteration where $\left[\mathbf{H}^{22}-\mathbf{A}^{22}\right]=\mathbf{G}_{\mathrm{w}}^{-1}-\mathbf{A}_{22}^{-1}$. However, due to different convergence in the iteration with different models, extra work may be required for the single-step method.
All the analyses of the results used the official 305-d lactation total yields of milk, protein, and fat (Lidauer et al., 2014). For validation of GEBV, the EBV for the lactation totals were obtained from the full data analysis. These were then deregressed for bull GEBV validation and cow GEBV validation using the Secant method in option DeRegress (Strandén and Mäntysaari, 2010) in MiX99 software. The effective record contribution (ERC) was used as a weighting factor in the deregressions. The ERC (Taskinen et al., 2013) were calculated by the ApaX99 program (Strandén et al., 2001) for all animals in the pedigree when full data was used. Variance parameters in ERC approximation were from the average daily TD model, and the same values $\left(\mathrm{h}_{\text {milk }}^{2}=0.48, \mathrm{~h}_{\text {protein }}^{2}=0.48\right.$, and $\left.\mathrm{h}_{\text {fat }}^{2}=0.49\right)$ were used throughout the study. Deregressions used the full pedigree in NAV evaluation and EBV for the bulls and cows from the full evaluation model. The 3 traits were deregressed simultaneously but assuming genetic and residual correlations to be zero. We chose to use DRP also for the bulls in the validation calculations instead of DYD to have directly comparable results with sire model GBLUP studies, including those in Koivula et al. (2012a).

Bulls born between years 2006 and 2009 and having their EBV based on ERC $\geq 3.0$ in the full data but having PA with $\mathrm{ERC}=0.0$ in the reduced data were defined as candidate bulls. For a bull, the phenotypic information obtained with $\mathrm{ERC}=3.0$ corresponds roughly to 20 daughters. For the cow validation, genotyped cows with no TD records in the reduced data and a minimum of $5 \mathrm{TD}$ records in the full data were considered candidate cows. Finally, in the validation test, we had 707 candidate bulls and 7,113 candidate cows. Validation reliability of predictions was assessed using the Interbull validation protocol (Mäntysaari et al., 2010):

$$
\mathbf{y}=\mathbf{1} \mathrm{b}_{0}+\mathrm{b}_{1} \hat{\mathbf{a}}+\mathbf{e},
$$

where $\mathbf{y}$ has the DRP of the candidate bulls or cows in the full data, $b_{0}$ and $b_{1}$ are unknown regression coefficients, â has the genomic prediction for bulls or cows based on the reduced data analysis (GEBV), and $\mathbf{e}$ is the residual error. The validation reliability of the model was obtained from the coefficient of determination $\left(\mathrm{R}^{2}\right)$ of the model $\left(\mathrm{R}_{\text {model }}^{2}\right)$, after correcting it by the average reliability of DRP $\left(\bar{r}_{D R P}^{2}\right)$ of the candidate bulls or cows; that is, $\mathrm{R}_{\text {validation }}^{2}=\mathrm{R}_{\text {model }}^{2} / \bar{r}_{D R P}^{2}$. The reliabilities of DRP were calculated as $r_{D R P}^{2}=E R C_{i} /\left(E R C_{i}+\lambda\right)$, where $\lambda=\left(1-\mathrm{h}^{2}\right) / \mathrm{h}^{2}$. To estimate the further gain from the genomic information over the traditional PA (VanRaden et al., 2009; Män- 
tysaari et al., 2010), the same validation tests were also applied to PA. Confidence intervals (CI) were estimated for the regression coefficients $\left(\mathbf{b}_{1}\right)$, the validation reliabilities, and the differences between the validation reliabilities among the model alternatives (e.g.,

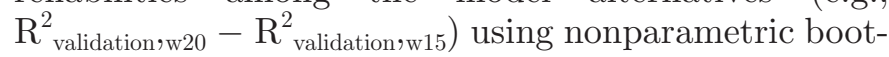
strap. The boot and boot.ci functions of the $\mathrm{R}$ package (R Development Core Team, 2012) were used to calculate the bootstrap CI separately for candidate bulls and cows. Number of bootstrap samples was 10,000. Bootstrap confidence intervals calculated using 3 methods: basic, norm, and perc. Confidence intervals by the basic method are given, because all methods gave approximately the same values.

\section{RESULTS AND DISCUSSION}

The number of iterations for EBV estimation was 3,818 , that for $\mathrm{EBV}_{\mathrm{r}}$ was 3,705 , and that for the ssGBLUP varied from 3,545 to 4,990 depending on the method used. The models were run into the same level of convergence: $\mathrm{C}_{\mathrm{a}}=(\sqrt{10.5})$, where $\mathrm{C}_{\mathrm{a}}=$ relative difference between left- and right-hand side of the part of the MME that includes the equations of the additive genetic animal effects. All models took about 35 to 54 $\mathrm{h}$ to run with 4 Intel Xeon $3.6 \mathrm{GHz}$ processors. There was an approximately $15 \%$ difference in iteration time: $33 \mathrm{~s}$ and, on average, $38 \mathrm{~s}$ per iteration round for EBV and ssGBLUP, respectively. Although the increase in computing time was mainly due to extra iterations, some differences were apparent between methods. According to the time used per iteration round, $\mathrm{w}_{20}$ and $\mathrm{w}_{15}$ had the best convergence among ssGBLUP methods. Computationally, the ssGBLUP added very little extra computing time to solving the mixed model equations by the PCG method. The only significant extra computations in the single-step method were due to the construction of the $\mathbf{H}^{22}$ matrix, which was done once before applying the PCG method. Presumably, the inclusion of genomic data affects convergence because the variance structure of genotyped animals in the $\mathbf{G}$ matrix is less diagonally dominant than with the pedigreebased relationship matrix $\mathbf{A}$ only. It has been shown that slight changes in scaling of $\mathbf{G}$ and $\mathbf{A}_{22}$ can affect convergence without a negative effect on validation accuracy (Meuwissen et al., 2011; Vitezica et al., 2011; Misztal et al., 2013). Generally, ssGBLUP with $\mathrm{w}_{15}$ or $\tau_{1.0} \omega_{0.5}$ needed the fewest iteration rounds to achieve convergence. Also, $\omega=0.5$ seemed to give better convergence than $\omega=0.6$ or $\omega=1.0$. This is in agreement with Misztal et al. (2013), who noted that larger $\omega$ causes $\mathbf{H}$ to be less positive definite, leading to slower convergence or even divergence when mixed model equations are solved by the iterative method, whereas smaller $\omega$ tend to give better convergence.

We also observed poor convergence with some $\mathbf{G}_{\mathrm{w}}$ matrices. Christensen et al. (2012) presented a method to scale and adjust the genomic relationship matrix. The proposed method calculates adjusted genomic relationship matrix $\mathbf{G}^{*}=a \mathbf{G}_{\mathrm{w}}+b \mathbf{1 1}^{\prime}$, where $\mathbf{1}$ is a vector of ones and the constants $a$ and $b$ are such that in $\mathbf{G}^{*}$, the average of diagonals and average of off-diagonals equal to those in the pedigree-based relationship matrix. We tested $\mathbf{G}^{*}$ in ssGBLUP using our data, but did not get solutions for further examination because the PCG algorithm showed poor convergence and failed to converge within 5,000 iterations. The adjustment constants for our data set were $a=0.968$ and $b=0.0323$; that is, the $\mathbf{G}_{\mathrm{w}}$ matrix was inflated by $3 \%$ after which all values were increased by a constant $b$. When the diagonal values of $\mathbf{G}_{\mathrm{w}}$ are $<1$, the $\mathbf{G}^{*}$ matrix is diagonally less dominant than $\mathbf{G}_{\mathrm{w}}$, which means increased correlations between animals and possibly poorer convergence of the PCG algorithm. There are other ways to correct genetic differences among genotyped and nongenotyped individuals (Vitezica et al., 2011; Lourenco et al., 2014).

Table 1 presents standard deviations of EBV and GEBV for reference and candidate bulls and cows in the full and reduced data sets. Standard deviations were derived within birth year and pooled across years to account for selection. For the reference animals, standard deviations of the GEBV closely followed standard deviations of the standardized EBV, although overall, some variation existed in the standard deviations of bulls and cows among different methods in building the $\mathbf{H}^{22}$ matrix. By using different $\mathrm{w}$, standard deviations of GEBV were close to that of EBV, whereas when using different $\tau$ and $\omega$, standard deviations of GEBV were somewhat lower than that of EBV. The reference bulls have a lot of progeny information and, there, changes in w did not affect reference animal GEBV much. In contrast, changes in $\tau$ and $\omega$ tended to have larger effects on reference bull standard deviations. In candidate bulls, standard deviations of GEBV increased with decreasing $\mathrm{w}$; that is, increased genomic information increased standard deviations of GEBV. Overall, in the candidate animals, the standard deviations of GEBV were higher than that of PA due to added information from genotypes. Standard deviations of the GEBV varied also with different estimation methods. In contrast to reference animals, in candidate bulls and cows, the use of different $\mathrm{w}$ gave higher standard deviations than the use of $\tau$ and $\omega$ in building the $\mathbf{H}^{22}$ matrix. In conclusion, the effect of changing $\tau$ and $\omega$ was an important one that affected standard deviations of both candidate 
Table 1. Standard deviations of EBV in the full data set and the reduced data $\left(\mathrm{EBV}_{\mathrm{r}}\right.$; for candidates, this is parent average, PA), and genomic enhanced breeding values with different single-step genomic BLUP (ssGBLUP) methods for the reference and candidate bulls and cows $^{1}$

\begin{tabular}{|c|c|c|c|c|c|c|}
\hline \multirow[b]{2}{*}{ Reference $^{2}$} & \multicolumn{2}{|c|}{ Milk } & \multicolumn{2}{|c|}{ Protein } & \multicolumn{2}{|c|}{ Fat } \\
\hline & Bulls & Cows & Bulls & Cows & Bulls & Cows \\
\hline No. of animals & 4,442 & 270 & & & & \\
\hline EBV & 11.11 & 8.83 & 10.51 & 7.97 & 11.52 & 10.27 \\
\hline $\begin{array}{l}\mathrm{EBV}_{\mathrm{r}} \\
\text { ssGBLUP }\end{array}$ & 10.93 & 9.42 & 10.39 & 9.21 & 11.40 & 11.71 \\
\hline $\mathrm{w}_{20}$ & 11.08 & 8.89 & 10.55 & 8.71 & 11.63 & 10.90 \\
\hline $\mathrm{w}_{15}$ & 11.08 & 8.81 & 10.54 & 8.62 & 11.63 & 10.80 \\
\hline $\mathrm{w}_{10}$ & 11.08 & 8.74 & 10.54 & 8.52 & 11.62 & 10.70 \\
\hline$\tau_{1.0} \omega_{0.5}$ & 10.57 & 7.79 & 9.99 & 7.62 & 11.08 & 9.61 \\
\hline$\tau_{1.6} \omega_{0.5}$ & 10.34 & 7.40 & 9.76 & 7.16 & 10.85 & 8.95 \\
\hline$\tau_{1.5} \omega_{0.6}$ & 10.44 & 7.56 & 9.87 & 7.31 & 10.96 & 8.17 \\
\hline$\tau_{1.6} \omega_{1.0}$ & 10.79 & 8.12 & 10.24 & 7.77 & 11.34 & 9.72 \\
\hline \multicolumn{7}{|l|}{ Candidate } \\
\hline No. of animals & 707 & 7,113 & & & & \\
\hline EBV & 10.85 & 9.19 & 10.12 & 9.02 & 10.07 & 9.87 \\
\hline $\begin{array}{l}\text { PA } \\
\text { ssGBLUP }\end{array}$ & 7.01 & 6.51 & 6.90 & 6.49 & 7.76 & 7.83 \\
\hline $\mathrm{w}_{20}$ & 8.93 & 8.92 & 8.90 & 8.86 & 9.50 & 10.52 \\
\hline $\mathrm{w}_{15}$ & 9.06 & 9.13 & 9.03 & 9.05 & 9.61 & 10.69 \\
\hline $\mathrm{w}_{10}$ & 9.20 & 9.37 & 9.17 & 9.26 & 9.73 & 10.87 \\
\hline$\tau_{1.0} \omega_{0.5}$ & 7.25 & 7.19 & 7.11 & 6.83 & 7.79 & 8.38 \\
\hline$\tau_{1.6} \omega_{0.5}$ & 7.26 & 7.26 & 7.07 & 6.81 & 7.66 & 8.24 \\
\hline$\tau_{1.5} \omega_{0.6}$ & 7.44 & 7.42 & 7.27 & 7.01 & 7.86 & 8.48 \\
\hline$\tau_{1.6} \omega_{1.0}$ & 8.73 & 8.87 & 8.62 & 8.60 & 9.10 & 10.09 \\
\hline
\end{tabular}

${ }^{1} \mathrm{EBV}$ are expressed as standardized EBV with SD of 10 units for all bulls and cows born between the years 1990 and 2012. Standard deviations were calculated within birth year and pooled across years.

${ }^{2} \mathrm{~W}=$ proportion of polygenic variance; $\tau=$ weight for $\mathbf{G}_{-1} ; \omega_{\mathrm{xx}}=$ weight for $\mathbf{A}_{22-1}$ matrix.

and reference animals when changes in w affected to a larger degree candidate animals.

The correlations between GEBV from different methods for candidate animals are presented in Table 2 . The correlations for reference bulls were all near to 1 (varying from 0.998 to 0.999 ). Thus, for proven bulls, different methods seem not to affect the GEBV greatly. For candidate bulls and cows, the correlations among different methods differed considerably, varying from 0.952 to 0.999 for bulls and from 0.933 to 0.999 for cows. There, the differences appeared between methods using $\mathrm{w}$ and methods using $\tau$ and $\omega$. The correlations between the different combinations of $\tau$ and $\omega$ increased as the amount of the additive genetic variance attributed to the genomic markers increased.

The model validation results for bulls are in Table 3 and for cows in Table 4. The tables present regression coefficients $\left(b_{1}\right)$ and validation reliabilities $\left(\mathrm{R}^{2}\right)$ with $95 \%$ bootstrap CI with 10,000 bootstrap resampling. For the bulls, validation reliabilities from the ssGBLUP with different $\mathbf{H}^{22}$ matrix block varied between 0.48 and 0.49 for milk, between 0.39 and 0.40 for protein, and between 0.41 and 0.44 for fat. The PA based on the same data but without genomic information gave, on average, 14-percentage-units lower reliability for milk, protein, and fat. For bulls, the best choice of param-

Table 2. Correlations (above diagonal = candidate bulls; below diagonal = candidate cows) among genomic enhanced breeding values from different methods in building genomic relationship matrices

\begin{tabular}{lcccccc}
\hline & \multicolumn{5}{c}{ Single-step genomic BLUP } \\
\cline { 2 - 7 } Method $^{1}$ & $\mathrm{w}_{20}$ & $\mathrm{w}_{15}$ & $\mathrm{w}_{10}$ & $\tau_{1.0} \omega_{0.5}$ & $\tau_{1.6} \omega_{0.5}$ & $\tau_{1.5} \omega_{0.6}$ \\
\hline $\mathrm{w}_{20}$ & & 0.999 & 0.996 & 0.957 & 0.952 & 0.961 \\
$\mathrm{w}_{15}$ & 0.998 & & 0.999 & 0.957 & 0.954 & 0.963 \\
$\mathrm{w}_{10}$ & 0.992 & 0.997 & & 0.957 & 0.956 & 0.965 \\
$\tau_{1.0} \omega_{0.5}$ & 0.933 & 0.940 & 0.947 & 0.996 & 0.999 \\
$\tau_{1.6} \omega_{0.5}$ & 0.933 & 0.942 & 0.950 & 0.996 & 0.989 & 0.992 \\
$\tau_{1.5} \omega_{0.6}$ & 0.944 & 0.952 & 0.960 & 0.996 & 0.999 & 0.974 \\
$\tau_{1.6} \omega_{1.0}$ & 0.982 & 0.989 & 0.993 & 0.957 & 0.967 & 0.975 \\
\hline
\end{tabular}

${ }_{\mathrm{W}}=$ proportion of polygenic variance; $\tau=$ weight for $\mathbf{G}_{-1} ; \omega_{\mathrm{xx}}=$ weight for $\mathbf{A}_{22-1}$ matrix. 
eters for milk was $\tau_{1.5} \omega_{0.6}$, and for protein and fat was $\tau_{1.6} \omega_{0.5}$. The lower $\omega$ for protein and fat means that more weight should be placed on genomic relationships for protein and fat relative to milk. For cows, validation reliabilities with different genomic matrix varied between 0.41 and 0.42 for milk, 0.31 and 0.33 for protein, and 0.32 and 0.34 for fat. Cow PA gave on average 12.6-percentage-unit lower reliabilities than GEBV. For cows, the best choice of parameters for milk and protein was $\tau_{1.5} \omega_{0.6}$, and for fat, the best choice was $\tau_{1.6} \omega_{0.5}$. Thus, protein has different results in cows compared with bulls. However, for cows, validation reliabilities can also differ because they have fewer close relatives in the reference population

Table 5 presents the root mean square errors (MSE) for the regressions. The MSE combine all criteria (inflation, trend, and accuracy), and the model with the lowest MSE should be the best one. Only small differences existed among MSE and they were mainly in line with the validation reliability results. However, according to MSE for bulls, the best parameter option for protein and fat would be $\tau_{1.6} \omega_{1.0}$, which contradicts the results from Table 3.

In general, our results indicate that differences in validation reliabilities among different methods are small, especially for bulls. This is demonstrated in more detail with the pairwise bootstrap comparisons, where only significant differences appear between validation reliabilities from PA and different GEBV, and between some ssGBLUP methods in fat (Table 6). For cows, significant differences seem to arise in validation reliabilities between methods using different values of $\mathrm{w}$, $\tau$, and $\omega$. Narrower CI could be achieved by increasing the number of validation animals. However, this would reduce the reference population size and could lead to lower validation reliability. Thus, significant differences in genomic prediction methods may be difficult to find when the number of genotyped animals is small. In addition, a small validation population has a large CI in validation reliability and this (random noise) may be one reason for different methods giving highest validation reliability, particularly in other studies where no confidence intervals have been given.

The degree of inflation is indicated by the coefficient of regression $\left(b_{1}\right)$ of true genetic values on $(G) E B V$. Optimal prediction of genetic merit of young individuals should have a regression coefficient of 1 . With $b_{1}$ $<1$, the predictions are inflated, and the differences in estimated genetic merit of young individuals are exaggerated compared with their future performance. For bulls, the $b_{1}$ values were almost always lower than the expected value, indicating that GEBV over-evaluate differences between bulls (Table 3). Changes in $\tau$ seemed to have only small effect on the $b_{1}$ value, unlike changes

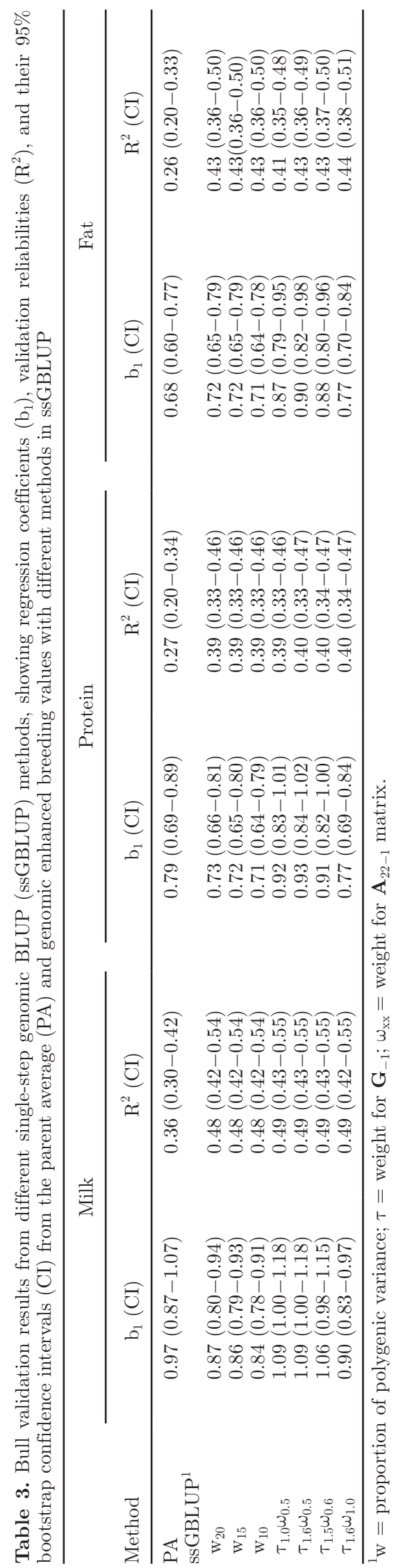




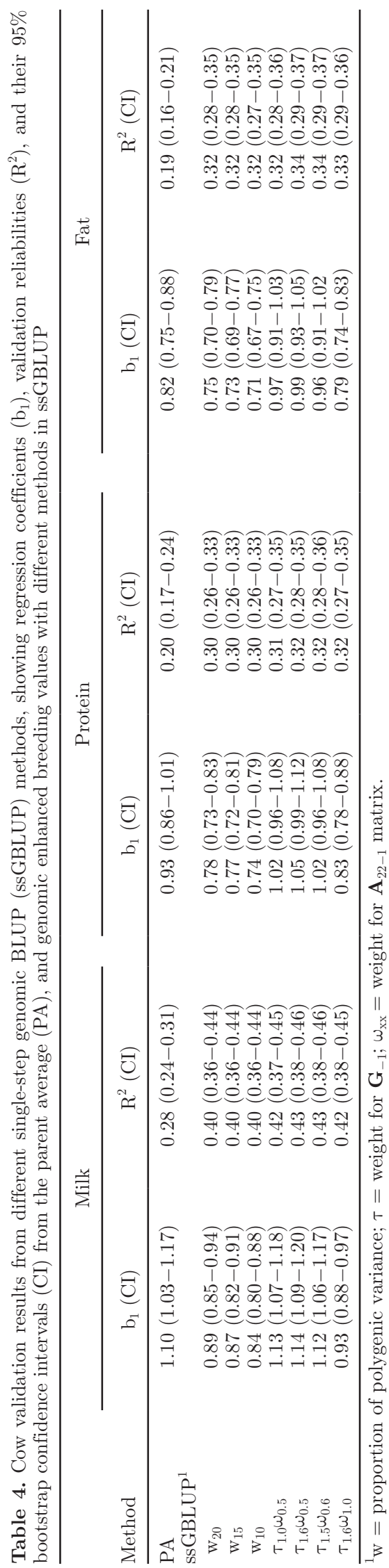

Table 5. Root mean square errors (MSE) from different validation regression models

\begin{tabular}{lccc}
\hline Model & Milk & Protein & Fat \\
\hline Bull & & & \\
$\mathrm{PA}$ & 644.81 & 20.94 & 25.77 \\
$\mathrm{~W}_{20}$ & 589.46 & 19.26 & 23.09 \\
$\mathrm{~W}_{15}$ & 589.46 & 19.26 & 23.00 \\
$\mathrm{~W}_{10}$ & 589.45 & 19.26 & 22.99 \\
$\tau_{1.0} \omega_{0.5}$ & 584.50 & 19.26 & 23.26 \\
$\tau_{1.6} \omega_{0.5}$ & 584.56 & 19.19 & 23.03 \\
$\tau_{1.5} \omega_{0.6}$ & 583.02 & 19.15 & 22.97 \\
$\tau_{1.6} \omega_{1.0}$ & 585.83 & 19.13 & 22.74 \\
Cow & & & \\
$\mathrm{PA}$ & 866.90 & 30.85 & 35.23 \\
$\mathrm{~W}_{20}$ & 840.04 & 30.19 & 34.17 \\
$\mathrm{~W}_{15}$ & 839.94 & 30.10 & 34.16 \\
$\mathrm{~W}_{10}$ & 840.24 & 30.20 & 34.17 \\
$\tau_{1 . .0} \omega_{0.5}$ & 836.96 & 31.00 & 34.09 \\
$\tau_{1.6} \omega_{0.5}$ & 835.20 & 30.05 & 34.02 \\
$\tau_{1.5} \omega_{0.6}$ & 834.56 & 30.04 & 34.01 \\
$\tau_{1.6} \omega_{1.0}$ & 836.28 & 30.08 & 34.17 \\
\hline
\end{tabular}

${ }^{1} \mathrm{PA}=$ parent average; $\mathrm{w}=$ proportion of polygenic variance; $\tau=$ weight for $\mathbf{G}_{-1} ; \omega_{\mathrm{xx}}=$ weight for $\mathbf{A}_{22-1}$ matrix.

in $\omega$. Decreasing $\omega$ increases weight of pedigree relationship matrix such that GEBV will be more influenced by pedigree information. Decrease in $\omega$ increased $b_{1}$ value. Only for milk and with $\tau_{1.6} \omega_{0.5}, \tau_{1.5} \omega_{0.5}$, or $\tau_{1.0} \omega_{0.5}$, the $b_{1}$ values were $>1$. However, depending on the method used to build the $\mathbf{H}^{22}$ matrix for ssGBLUP, the overdispersion was very similar to or even lower than that with PA. This suggests that GEBV by the single-step method are less biased than PA, but it is still essential to determine the best method to build the $\mathbf{H}^{22}$ matrix. The results were similar with cows, although in general, $b_{1}$ values were higher for cows than for bulls, indicating a smaller bias (Table 4). Several studies have found better accuracies and lower biases of GEBV by fine-tuning $\mathrm{w}, \tau$, and $\omega$ when constructing the $\mathbf{H}$ matrix (Misztal et al., 2013). Moreover, validation reliability has been observed to be less sensitive to changes in $\mathrm{w}$ than accuracy (Christensen et al., 2012).

The genetic trends in milk (G)EBV for genotyped bulls are shown in Figure 1. For reference bulls, the genetic trends with different GEBV followed close to the $\mathrm{EBV}$ and $\mathrm{EBV}_{\mathrm{r}}$ trends, although there was a tendency for methods using $\mathrm{w}$ to give genetic trends that were closer to EBV compared with methods using a wider range of $\tau$ and $\omega$. For the candidate bulls, the ssGBLUP with different $\mathrm{w}$ as well as PA seem to overestimate the trend, whereas methods using $\tau$ and $\omega$ seemed to underestimate the trend. Figure 1 indicates that putting more weight on genomic information is desirable. However, this must be balanced with the proper parameter choice for $\tau$ and $\omega$ to have the correct combination of genomic and pedigree relationships. 
Table 6. Pairwise comparisons of validation reliabilities $\left(\mathrm{R}^{2}\right)$ from different methods in single-step genomic BLUP (ssGBLUP) and parent average $(\mathrm{PA})^{1}$

\begin{tabular}{|c|c|c|c|c|c|c|c|c|}
\hline Method & $\mathrm{PA}$ & $\mathrm{w}_{20}$ & $\mathrm{w}_{15}$ & $\mathrm{w}_{10}$ & $\tau_{1.0} \omega_{0.5}$ & $\tau_{1.6} \omega_{0.5}$ & $\tau_{1.5} \omega_{0.6}$ & $\tau_{1.6} \omega_{1.0}$ \\
\hline $\mathrm{w}_{20}$ & M P F & & & & & & & $\mathrm{F}$ \\
\hline$\tau_{1.0} \omega_{0.5}$ & M P F & & & & & $\mathrm{F}$ & $\mathrm{F}$ & $\mathrm{F}$ \\
\hline$\tau_{1.6} \omega_{0.5}$ & M P F & M P F & M P F & M P F & M P F & & $\mathrm{F}$ & \\
\hline$\tau_{1.5} \omega_{0.6}$ & M P F & M P F & M P F & M P F & M P F & M & & \\
\hline
\end{tabular}

${ }^{1}$ Letters indicate significant differences between compared methods. Differences in methods for bulls are shown above the diagonal and those for cows below the diagonal; M, P, F indicate milk, protein, and fat.

${ }^{2} \mathrm{~W}=$ proportion of polygenic variance; $\tau$ = weight for $\mathbf{G}_{-1} ; \omega_{\mathrm{xx}}=$ weight for $\mathbf{A}_{22-1}$ matrix.

Comparison of different methods to build the $\mathbf{H}^{22}$ matrix for the ssGBLUP showed that alternative parameters could be chosen depending on whether the goal is to achieve higher validation reliabilities or smaller bias. In general, differences in validation reli- abilities and their CI (Table 3 and Table 4) with the studied methods were smaller than effects to regression coefficient $b_{1}$, which indicate bias. Therefore, it is essential to consider the whole picture before choosing which method to use. Moreover, different methods can

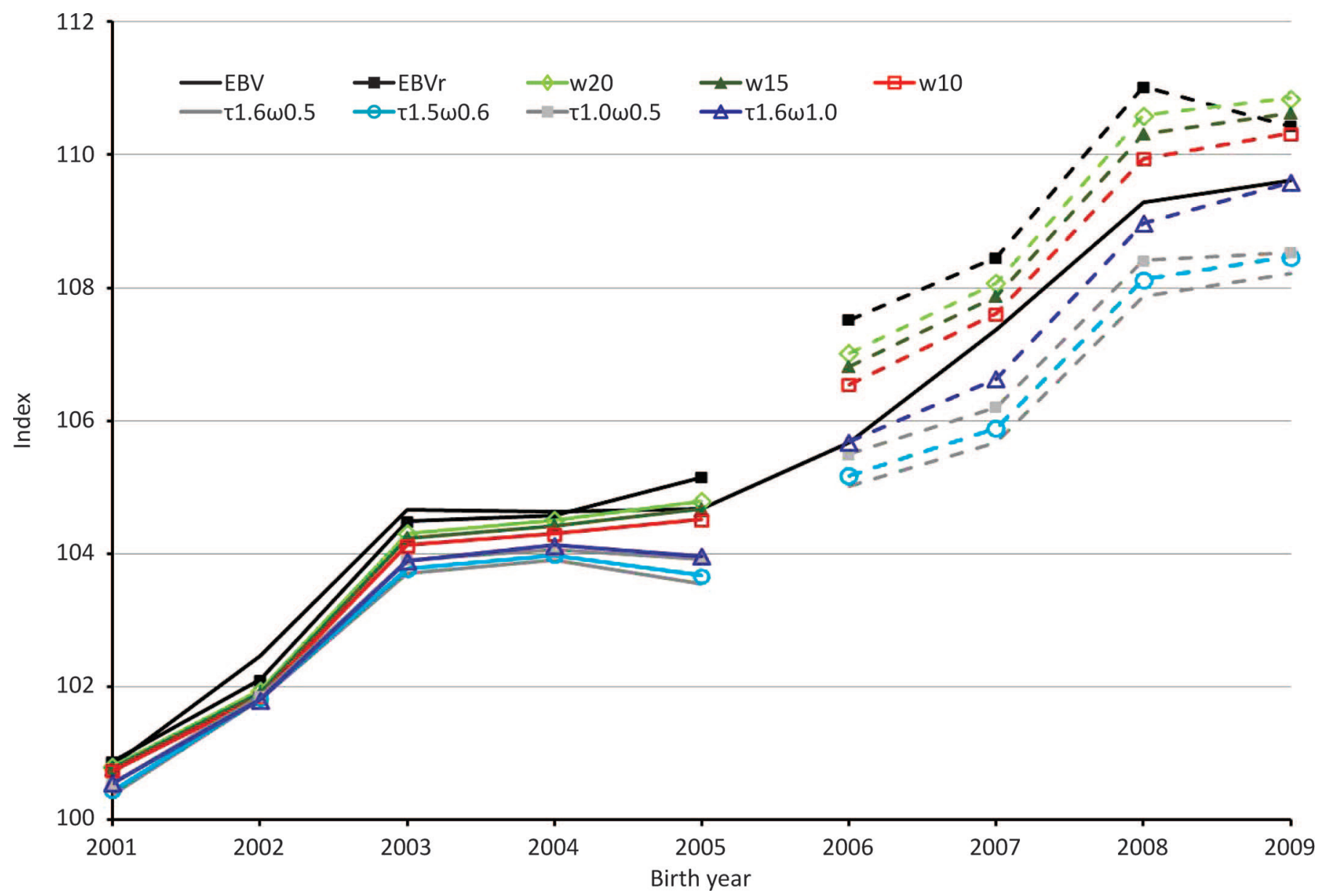

Figure 1. Genetic trends for milk genomic enhanced breeding values (GEBV) and EBV of reference and candidate bulls from reduced data (i.e., $\mathrm{EBV}_{\mathrm{r}}$ ) with different methods used in single-step genomic BLUP (ssGBLUP). For the candidate bulls, the $\mathrm{EBV}_{\mathrm{r}}$ is parent average (PA); EBV are from the full test-day model. Solid lines indicate reference bulls and dashed lines indicate candidate bulls. $\mathrm{w}=$ proportion of polygenic variance; $\tau=$ weight for $\mathbf{G}_{-1} ; \omega_{\mathrm{xx}}=$ weight for $\mathbf{A}_{22-1}$ matrix. Color version available online. 
be optimal for different traits. Differences in optimal weighting factors may be due to differences in genetic architecture. Use of a genomic relationship matrix that weights markers according to analyzed trait (e.g., VanRaden, 2008; Makgahlela et al., 2013) may better account for differences in genetic architecture.

Validation reliabilities for bulls from the current study were generally higher than the validation reliabilities obtained for RDC with genomic evaluation by multi-step approach and by sire model single-step method (Koivula et al., 2012a; Su et al., 2012). This is partly due to the larger reference population. It also appears that we achieved better validation reliabilities by using phenotypic data in the ssGBLUP than by using deregressed breeding values (DRP) from either sire or animal model deregression (Mäntysaari et al., 2011; Koivula et al., 2012a,b, 2014). Moreover, based on the regression coefficients $b_{1}$, the GEBV from the phenotypic records seemed to be less inflated than the DGV from sire model or GEBV from animal model deregressions. Still, the models in the current study might fail the Interbull GEBV validation test. From bootstrapping with 10,000 samples, CI for the regression coefficients of the GEBV reached 1.0 with only some methods in milk and protein; for fat, the upper limits were always $<1.0$. The bootstrap CI are consistent with results in Mäntysaari and Koivula (2012), in which we suggested that to reach a standard error of 0.049 for the estimate of $b_{1}$ requires 443 bulls. However, the Interbull requirement for unbiasedness will accept $b_{1}$ estimates between 0.90 and 1.20 , even if the CI of $b_{1}$ does not include 1. Thus, $a b_{1}$ estimate of 0.9 is not considered biologically significantly biased. Therefore, a standard error of 0.05 is an important limit for the power of the test, because with it the statistical and biological significance agree. Reversing the power consideration, we could suggest that the number of validation bulls should always be at least 500 .

The ssGBLUP has been used for several large-scale analyses, including in dairy cattle (Aguilar et al., 2011; Tsuruta et al., 2011; VanRaden, 2012), pigs (Forni et al., 2011; Christensen et al., 2012), and broiler chickens (Chen et al., 2011). Experiences from these studies have indicated that ssGBLUP gives as high or higher validation reliability than multi-step methods, and the inflation of GEBV is generally smaller. Another reason for using ssGBLUP is the ability to account for selection bias when selection is based on genotypes only (VanRaden, 2012).

The current study shows that ssGBLUP is easy to implement in existing national evaluation models. In this way, phenotypic records are combined directly with genomic information and, thus, resulting GEBV directly combine both sources of information. Moreover, in
TD ssGBLUP, genomic information can be accounted for in estimation of environmental effects. Additional computational costs in the single-step approach may be lower than in multiple-step genomic evaluations. In our study, the number of genotyped bulls was relatively low, and the $\mathbf{G}$ matrix was easy to invert. For a large number of genotyped animals, algorithms have been proposed that overcome the need of $\mathbf{G}$ inverse, for example, by Ducrocq and Legarra (2011), Legarra and Ducrocq (2012), and Liu et al. (2013). This could also make ssGBLUP computationally more appealing. However, it may also cause convergence problems in iterative methods (Strandén and Mäntysaari, 2014).

\section{CONCLUSIONS}

Our results show that the use of phenotypic test-day records in single step analysis is feasible. The ssGBLUP provides a good alternative to the current multi-step approach used for Nordic RDC. The ssGBLUP is easy to implement and it gives results comparable to those of the original models. Moreover, the use of phenotypic records gave higher validation reliabilities compared with earlier validations that used sire model or animal model deregressions. However, it is essential to find the optimal way to combine $\mathbf{G}$ and $\mathbf{A}$ matrixes to minimize bias and maximize reliability of GEBV.

\section{ACKNOWLEDGMENTS}

This work was a part of the Genomic Selection project originally established by Aarhus University and the Nordic cattle breeding organizations Viking Genetics (Randers, Denmark), Nordic Cattle Genetic Evaluation (Aarhus, Denmark), and Faba (Hollola, Finland). They are acknowledged for providing the genotype and testday data.

\section{REFERENCES}

Aguilar, I., I. Misztal, D. L. Johnson, A. Legarra, S. Tsuruta, and T. J. Lawlor. 2010. Hot topic: A unified approach to utilize phenotypic, full pedigree, and genomic information for genetic evaluation of Holstein final score. J. Dairy Sci. 93:743-752.

Aguilar, I., I. Misztal, S. Tsuruta, G. R. Wiggans, and T. J. Lawlor. 2011. Multiple trait genomic evaluation of conception rate in Holsteins. J. Dairy Sci. 94:2621-2624.

Chen, C. Y., I. Misztal, I. Aguilar, S. Tsuruta, T. H. E. Meuwissen, S. E. Aggrey, T. Wing, and W. M. Muir. 2011. Genome-wide markerassisted selection combining all pedigree phenotypic information and genotypic data in one step: An example using broiler chickens. J. Anim. Sci. 89:23-28.

Christensen, O. F., and M. S. Lund. 2010. Genomic prediction when some animals are not genotyped. Genet. Sel. Evol. 42:2.

Christensen, O. F., P. Madsen, B. Nielsen, T. Ostersen, and G. Su. 2012. Single-step methods for genomic evaluation in pigs. Animal $6: 1565-1571$. 
Ducrocq, V., and A. Legarra. 2011. An iterative implementation of the single step approach for genomic evaluation which preserves existing genetic evaluation models and software. Interbull Bull. 44:138-142.

Forni, S., I. Aguilar, and I. Misztal. 2011. Different genomic relationship matrices for single-step analysis using phenotypic, pedigree and genomic information. Genet. Sel. Evol. 43:1.

Hayes, B. J., P. J. Bowman, A. J. Chamberlain, and M. E. Goddard. 2009. Invited review Genomic selection in dairy cattle: Progress and challenges. J. Dairy Sci. 92:433-443.

Koivula, M., I. Strandén, G. P. Aamand, and E. A. Mäntysaari. 2014. Effect of cow reference group on validation accuracy of genomic evaluation. Proc. 10th World Congr. Genet. Appl. Livest. Prod., Vancouver, Canada, Aug. 17-22, Comm. 083.

Koivula, M., I. Strandén, J. Pösö, G. P. Aamand, and E. A. Mäntysaari. 2012b. Single step genomic evaluations for the Nordic Red Dairy cattle test day data. Interbull Bull. 46:115-120.

Koivula, M., I. Strandén, G. Su, and E. A. Mäntysaari. 2012a. Different methods to calculate genomic predictions - Comparisons of SNP-BLUP, G-BLUP and H-BLUP. J. Dairy Sci. 95:4065-4073.

Legarra, A., I. Aguilar, and I. Misztal. 2009. A relationship matrix including full pedigree and genomic information. J. Dairy Sci. 92:4656-4663.

Legarra, A., and V. Ducrocq. 2012. Computational strategies for national integration of phenotypic, genomic, and pedigree data in a single-step best linear unbiased prediction. J. Dairy Sci. 95:46294645 .

Lidauer, M. H., J. Pösö, J. Pederson, J. Lassen, P. Madsen, E. A. Mäntysaari, U. Nielsen, J.-A. Eriksson, K. Johansson, T. Pitkänen, I. Strandén, and G. P. Aamand. 2015. Across-country test-day model evaluations for Nordic Holstein, Red Cattle and Jersey. J. Dairy Sci. 98:1296-1309. http://dx.doi.org/10.3168/jds.2014-8307.

Liu, Z., M. Goddard, F. Reinhardt, and R. Reents. 2013. Computing strategies for a single step SNP model with an across country reference population. No. 19:452 in Book of Abstracts: 64th Annu. Mtg. EAAP, Nantes, France. EAAP, Rome, Italy.

Lourenco, D. A. L., I. Misztal, S. Tsuruta, I. Aguilar, T. J. Lawlor, S. Forni, and J. I. Weller. 2014. Are evaluations on young genotyped animals benefiting from the past generations? J. Dairy Sci. 97:3930-3942.

Makgahlela, M., T. Knürr, G. P. Aamand, I. Strandén, and E. A. Mäntysaari. 2013. Single step evaluations using haplotype segments. Interbull Bull. 47:217-221.

Mäntysaari, E., Z. Liu, and P. VanRaden. 2010. Interbull validation test for genomic evaluations. Interbull Bull. 41:17-24.

Mäntysaari, E. A., and M. Koivula. 2012. GEBV validation test revisited. Interbull Bull. 45:11-16.

Mäntysaari, E. A., M. Koivula, I. Strandén, J. Pösö, and G. P. Aamand. 2011. Estimation of GEBVs using deregressed individual cow breeding values. Interbull Bull. 44:19-24.
Meuwissen, T. H. E., T. Luan, and J. A. Woolliams. 2011. The unified approach to the use of genomic and pedigree information in genomic evaluations revisited. J. Anim. Breed. Genet. 128:429-439.

Misztal, I., I. Aguilar, A. Legarra, and T. J. Lawlor. 2010. Choice of parameters for single-step genomic evaluation for type. J. Dairy Sci. 93(Suppl. 1):533. (Abstr.)

Misztal, I., A. Legarra, and I. Aguilar. 2009. Computing procedures for genetic evaluation including phenotypic, full pedigree, and genomic information. J. Dairy Sci. 92:4648-4655.

Misztal, I., S. Tsuruta, I. Aguilar, A. Legarra, P. M. VanRaden, and T. J. Lawlor. 2013. Methods to approximate reliabilities in single-step genomic evaluation . J. Dairy Sci. 96:647-654.

R Core Development Team. 2012. R: A language and environment for statistical computing. R Foundation for Statistical Computing, Vienna, Austria. http://www.R-project.org/.

Strandén, I., and M. Lidauer. 1999. Solving large mixed models using preconditioned conjugate gradient iteration. J. Dairy Sci. 82:2779-2787.

Strandén, I., M. Lidauer, E. A. Mäntysaari, and J. Pösö. 2001. Calculation of Interbull weighting factors for the Finnish test day model. Interbull Bull. 26:78-81.

Strandén, I., and E. A. Mäntysaari. 2010. A recipe for multiple trait deregression. Interbull Bull. 42:21-24.

Strandén, I., and E. A. Mäntysaari. 2014. Comparison of some equivalent equations to solve single-step GBLUP. Proc. 10th World Congr. Genet. Appl. Livest. Prod., Vancouver, Canada, Aug. 17-22, Comm. 069

Su, G., P. Madsen, U. S. Nielsen, E. A. Mäntysaari, G. P. Aamand, O. F. Christensen, and M. S. Lund. 2012. Genomic prediction for the Nordic Red Cattle using one-step and selection index blending approaches. J. Dairy Sci. 95:909-917.

Taskinen, M., E. Mäntysaari, M. Lidauer, T. Knürr, J. Pösö, G. Su, G. P. Aamand, and I. Strandén. 2013. Comparison of model reliabilities from single-step and bivariate blending methods. Interbull Bull. 47:246-251.

Tsuruta, S., I. Misztal, I. Aguilar, and T. J. Lawlor. 2011. Multipletrait genomic evaluation of linear type traits using genomic and phenotypic data in US Holsteins. J. Dairy Sci. 94:4198-4204.

VanRaden, P. M. 2008. Efficient methods to compute genomic predictions. J. Dairy Sci. 91:4414-4423.

VanRaden, P. M. 2012. Avoiding bias from genomic pre-selection in converting daughter information across countries. Interbull Bull. 45:1-5.

VanRaden, P. M., C. P. Van Tassell, G. R. Wiggans, T. S. Sonstegard, and R. D. Schnabel. 2009. Invited review: Reliability of genomic predictions for North American Holstein bulls. J. Dairy Sci. 92:16-24.

Vitezica, Z. G., I. Aguilar, I. Misztal, and A. Legarra. 2011. Bias in genomic predictions for populations under selection. Genet. Res. (Camb.) 93:357-366. 